\title{
The Work of Health Educators in a Clinical Setting
}

\author{
Lisa Smith and James F. McKenzie \\ Ball State University
}

\begin{abstract}
This study examined the task of health educators working in a clinical setting to better understand their roles. The study was designed as a descriptive, cross-sectional survey using a convenience sample of health educators who worked in clinical settings in Indiana and who had a college degree in health education. Forty health educators were contacted and 62\% of the subjects participated in the survey. Results indicated most jobs were similar in nature and the health education curriculum studied helped them in the roles they were serving. However, many educators felt there were other content areas of study that would have been helpful to prepare them for working in the clinical setting. A majority of the respondents reported that having Certified Health Education Specialist (CHES) certification was helpful and necessary in their positions.
\end{abstract}

(C) 2006 Californian Journal of Health Promotion. All rights reserved.

Keywords: Health educators, health education, clinical setting, public health

\section{Introduction}

The process to define the role of a health educator began in 1978. By 1981, the generic role was defined. Shortly thereafter came the writing of the Competency-Based Framework for the Professional Development of Certified Health Education Specialist (National Commission for Health Education Credentialing, Inc. [NCHEC], 1996). Recent challenges in public health since September 11, 2001, have highlighted the role of health educators and research has described its supply, geographic distribution, educational preparation and desired competencies (Finacchio, Love, \& Sanchez, 2003).

Even though there appears to be a need for health educators, and we profess to prepare generic health educators, some have questioned whether it is possible to do so because of the peculiarities of each setting (McKenzie, 2004). Examples of such peculiarities include the culture in the business world (work site setting), and the language used in health care (clinical setting). Because of some of these peculiarities, it has been very difficult for health educators to secure health education positions in the clinical setting. Employers have been more inclined to hire individuals with clinical training than health educators for health education positions in the clinical setting. While the work of health educators in community health settings is well understood, very little information has been written on this subject of health educators working in the clinical setting. Therefore, the purpose of this study is to find out more about the work of health educators in the clinical setting. This information would not only be helpful to new health educators seeking employment in a clinical setting, but also to healthcare organizations that could benefit from employing health educators.

\section{Methods}

\section{Participants}

The participants in this study were selected using a snowball sample. A snowball sample includes those identified by the researchers and others referred by initial participants (McKenzie, Neiger, \& Smeltzer, 2005). In this study the researchers identified initially 20 potential participants. These individuals were called and invited to participate in the study. If they agreed, they were interviewed. At the conclusion of the interview, they were asked to give the name(s) of other health educator(s) working in Indiana in 
a clinical setting, thus, the snowballing of the sample. A total of 40 individuals were contacted using this process with 25 (62.5\%) willing to participate.

\section{Instrumentation}

An original questionnaire was created to identify the roles and responsibilities of health educators from Indiana working in clinical settings. Perhaps the most challenging aspect of developing a useful instrument was deciding what information was needed to better understand health educators and their roles in the clinical setting. The researchers developed an initial pool of 20 questions for the instrument. A review of the questions by her advisor was used to establish face validity. Content validity was established using a jury of experts. The jury was composed of six health educators working in a clinical setting. After the jurors agreed to participate, they were emailed a letter explaining the purpose of the instrument, a draft of the instrument and instructions for completing three tasks. First, they were asked to read each question and to make suggestions for making any unclear questions better. Second, they were asked to offer suggestions or new questions they thought should be included in the instrument. And third, each was asked to rate the questions as essential, useful but not essential, or not necessary. Upon receipt of the jurors' work, unclear items were rewritten, and some questions were deleted and added. The final instrument contained 24 questions.

\section{Data Collection}

Data were collected via a telephone or email survey. When the subjects were contacted by telephone, they were asked if they would be willing to participate in a survey about health educators working in a clinical setting. If they agreed to participate, they were interviewed at that time or at another agreed-upon convenient time. If the subject felt uncomfortable about being interviewed by telephone, or did not have time for a telephone interview, the questionnaire was emailed to them for completion. Of the 25 completed questionnaires, two (8\%) were completed via telephone and 23 (92\%) were completed via email.
Upon receipt of each completed questionnaire, each was coded and the data were transferred to a computer scan form. Data analysis included tabulation of frequencies and percentages.

\section{Results}

Sixty-five point five percent $(n=25)$ of health educators who were invited to participate in the study did so by either answering the questionnaire verbally over the telephone or completing a questionnaire and returning it by email. These 25 individuals worked for 12 different agencies. Of the agencies and organizations employing the participants in the study, most $76 \%(n=19)$ of these agencies were not-for-profit organizations. Of those who participated, 98\% $(n=23)$ were females and $44 \%$ $(n=11)$ were CHES certified. Another 16\% $(n=4)$ reported to have taken the exam and were awaiting results at the time this survey was conducted.

When participants were asked about their job title, 15 different job titles were given (see Appendix A). Sixty-eight percent of the respondents $(n=27)$ reported the number of health educators on staff was between one and five. When the respondents were asked about their major job responsibilities, community education was the most reported response, followed by management, teaching nutrition/ fitness, patient education, scheduling, program planning, coordination of planning, being a community liaison, and supervision, (see Appendix B).

A number of questions were asked about the services provided by the health educators and their agencies. Eighty-four percent $(n=21)$ offered public education. Seventy-two percent $(n=18)$ offered employee education, and 64\% $(n=16)$ offered patient education programs. There were $88 \%(n=22)$ who reported attending health fairs, with $72 \%(n=18)$ who attended less than 19 per year. When asked if they charged for health education programs, 64\%, $(n=16)$ did not charge. Yet, 68\% $(n=17)$ of the respondents reported they relied on grant dollars for program funding. Of those who relied on grant funding, $28 \%(n=7)$ of the respondents reported $90-100 \%$ of their budget was grant funded. 
When asked with what agencies they worked most closely, $44 \% \quad(n=11)$ worked with voluntary agencies, 36\% ( $\mathrm{n}=9)$ worked with schools, 36\% (n=9) work with local health departments, $16 \% \quad(n=4)$ work with county tobacco coalitions, $12 \%(n=3)$ with hospitals, state health departments, churches or religious organizations, and health centers, $8 \%(n=2)$ with state agencies, and less that $4 \%(n=1)$ worked with city government, centers for aging, YMCA, community agencies and federal agencies.

One hundred percent of the respondents reported offering activities outside the clinical setting (also known as outreach programs). In these outreach programs, $80 \%(\mathrm{n}=20)$ used printed materials, $76 \% \quad(n=19)$ offered educational programs, and $60 \%(n=15)$ offered free health screenings. Most respondents $(80 \%, n=32)$ reported that their agency paid for materials instead of getting donations for materials or creating their educational materials in-house.

Several questions were asked about the Certified Health Education Specialist (CHES) credential. Most of the respondents, $72 \%(n=18)$ felt the CHES certification was helpful in their positions. There were $56 \%(n=14)$ who said the CHES certification was necessary to sell themselves to potential employers. Only $16 \%$ $(n=4)$ of the respondents felt a CHES certification was not necessary in their positions.

Finally, the participants were asked about salary. The most commonly reported salary range of a health educator was between $\$ 25,000$ and $\$ 35,000$. Of course, this would depend on levels of experience and education.

\section{Discussion}

As hypothesized, more clinical skills are needed to secure health education positions in the clinical setting. Most respondents felt a CHES certification was beneficial to their positions. When asked what heath educators could do to "sell" themselves to prospective employers, 60\% $(n=15)$ said demonstrating knowledge of health education, program planning, program implementation, evaluation and grant writing were the best skills to have to sell themselves. In addition, being able to communicate or explain what health educators can do for a company was answered by $24 \%(n=6)$ of the respondents. Twelve percent $(n=3)$ said to research the company and find out how you can meet their needs. One respondent (4\%) suggested taking examples of work done from internships and classes such as brochures, PowerPoint ${ }^{\circledR}$ presentations, display boards, and other works to show the prospective employer. When asked about other course work they would recommend in terms of academic preparation to be a health educator in a clinical setting, $24 \%(n=6)$ said clinical physiological testing skills were important to know. Twelve percent $(n=3)$ said volunteer experience in a clinical setting was important, marketing and sales courses, communication classes and education courses would be helpful. Counseling or social work courses, medical terminology and nutrition courses were the answer given by $8 \%(n=2)$ of the respondents of courses helpful in preparation of a career as health educators.

What do these results mean for the profession of health education? If upon graduation, health education students want to work in the clinical setting, they must make good choices on how to choose their elective courses. The courses they choose should emphasize clinical skills, communication, education, marketing, counseling, medical terminology, and nutrition. They should also have volunteer experience in a clinical setting, and choose to complete an internship in a clinical setting. After graduation, when trying to gain employment in a clinical setting, they need to be able to demonstrate the knowledge they possess, the experiences they have gained and demonstrate what they can offer to prospective employers. Many employers do not know very much about health education graduates and need to be educated to the benefits of hiring a health educator to fill clinical positions.

This study was not without its limitations. The greatest limitation was the selection of the participants. The snowball sampling technique was used because resources were limited and a probability sample was too costly and time consuming to complete. Non-probability samples have limitations because results can 
only be generalized to those who participated. Bias may occur since those who are not included in the sample may differ in ways from those who are included in the sample (McKenzie et al., 2005).

\section{Conclusions}

In conclusion, additional studies on health educators working in the clinical setting need to be completed to gain a better understanding of the profession. Leaders of organizations who employ people in clinical settings need to have a better understanding of what health educators are doing and the potential they can bring to an organization. In order to change the mind-set of many who feel that only those with clinical training should do patient education creates a great obstacle, which needs to be overcome. Only with more information available and continued monitoring of credentialing to provide a set of standards for health educators, can attitudes be changed and gains be made in the area of health educators working in a clinical setting.

\title{
References
}

Benz-Scott, L., \& Black, D. (1999). Health communication and professional preparation: Health educator credibility, message learning, and behavior change. Health Education \& Behavior, 26, 609-620.

Finacchio, L., Love, M., \& Sanchez, E. (2003). Illuminating the MPH health education workforce: Results and implications of an employer survey. Health Education and Behavior, 30, 683-693.

Lamarine, R., Fisher, J., \& Sbarbaro, V. (2003). Attitudes and practices of university health education faculty related to alternative medicine. Californian Journal of Health Promotion, 1(3), 105-122.

McKenzie, J. F. (2004). Professional preparation: Is a generic health educator really possible? American Journal of Health Education, 35(1), 46-48.

McKenzie, J. F., Neiger, B. L., \& Smeltzer, J. L. (2005). Planning, implementing and evaluating health promotion programs: A primer (4th ed.). San Francisco, CA: Benjamin Cummings.

McKenzie, J. F., \& Smeltzer, J. L. (2001). Planning, implementing and evaluating health promotion programs: A primer (3rd ed.). Needham Heights, MA: Allyn and Bacon.

National Commission for Health Education Credentialing, Inc. (1996). A competency-based framework for professional development of certified health education specialists. Allentown, PA: Author.

Timmerick, T. (2003). Planning, program development and evaluations (2nd ed.). Sudbury, MA: Jones and Bartlett Publishers.

\author{
Author Information \\ Lisa Smith, B.S. \\ E-Mail: lisaannsmith03@yahoo.com \\ James F. McKenzie, PhD, M.P.H., CHES* \\ Department of Physiology and Health Science \\ Ball State University \\ Ph. 765-285-8345 \\ E-Mail: jmckenzie@bsu.edu \\ * corresponding author
}




\section{Appendix A}

Job Titles of the Participants

\begin{tabular}{|l|c|}
\hline \multicolumn{1}{|c|}{ Job Title } & Number/Percent \\
\hline Director & $4(16 \%)$ \\
\hline Program Manager & $3(12 \%)$ \\
\hline Health Educator & $3(12 \%)$ \\
\hline Account Executive & $2(8 \%)$ \\
\hline Wellness Coordinator & $2(8 \%)$ \\
\hline Assistant Director & $1(4 \%)$ \\
\hline Program Coordinator & $1(4 \%)$ \\
\hline Patient Education Coordinator & $1(4 \%)$ \\
\hline Community Health Educator & $1(4 \%)$ \\
\hline Exercise Leader & $1(4 \%)$ \\
\hline Screening Specialist & $1(4 \%)$ \\
\hline Chronic Disease Specialist & $1(4 \%)$ \\
\hline Preventive Disease Specialist & $1(4 \%)$ \\
\hline Manager & $1(4 \%)$ \\
\hline School Health Program Director & $1(4 \%)$ \\
\hline Left Blank & $1(4 \%)$ \\
\hline
\end{tabular}




\section{Appendix B}

Major Job Responsibilities

\begin{tabular}{|l|c|}
\hline \multicolumn{1}{|c|}{ Job Responsibilities } & Number/Percent \\
\hline Education & $7(28 \%)$ \\
\hline Patient Education & $4(16 \%)$ \\
\hline Management & $4(16 \%)$ \\
\hline Evaluation & $4(16 \%)$ \\
\hline Program Planning & $3(12 \%)$ \\
\hline Scheduling & $3(12 \%)$ \\
\hline Community Education & $3(12 \%)$ \\
\hline Teaching nutrition & $3(12 \%)$ \\
\hline Coordination of programs & $2(8 \%)$ \\
\hline Community Liaisons & $2(8 \%)$ \\
\hline Training & $2(8 \%)$ \\
\hline Bulletin/ Display boards & $2(8 \%)$ \\
\hline Supervision & $2(8 \%)$ \\
\hline Employee health promotion & $2(8 \%)$ \\
\hline Grant Reporting & $1(4 \%)$ \\
\hline Program Development & $1(4 \%)$ \\
\hline Processing Paper work & $1(4 \%)$ \\
\hline Fund-raising & $1(4 \%)$ \\
\hline Work Site Wellness & $1(4 \%)$ \\
\hline Program Implementation & $1(4 \%)$ \\
\hline Conduction of Meetings & $1(4 \%)$ \\
\hline Administering follow up programs & $1(4 \%)$ \\
\hline Grant Writing & $1(4 \%)$ \\
\hline Collaboration & $1(4 \%)$ \\
\hline Program Policy & $1(4 \%)$ \\
\hline Consultations & $1(4 \%)$ \\
\hline Problem Solving & $1(4 \%)$ \\
\hline Blank & $5(20 \%)$ \\
\hline
\end{tabular}

